\title{
Study on the Influence of Weld Defects on Structural Vibration Responses
}

\author{
Lizong Lin*, Chengxiao Yang \\ East China University of Science and Technology, China \\ *Izlin@ecust.edu.cn
}

\begin{abstract}
Keywords: welding defects; cross correlation function amplitude vector (CorV); numeric simulation; vibration response
\end{abstract}

\begin{abstract}
In this article we study the influence of weld defects on vibrating responses using a simplified numerical model--flat butt plate with a type-I welded joint. To analyze the changes in vibrating responses influenced by weld defects, a method based on cross correlation function amplitude vector (CorV) is adopted. The relative change of CorV is selected as a criterion for discriminating defects. The numeric results show that both single and multiple defects have a great influence on the criterion and also verify that the potential of the CorV based on acceleration responses for detecting and locating welding defects. We simulate the severity of defects by $10-80 \%$ reductions in modulus of elasticity, and make the conclusion that the relative change in CorV increases as the reduction occurs.
\end{abstract}

\section{Introduction}

As a kind of processing technology, welding technology, which can reflect the level of national industrialization and scientific modernization, is widely applied to various large structural components. The welding process is characterized by physicochemical changes which inevitably lead to the non-uniformity of performance of welded joints and complex stress distribution. In order to obtain a reliable welded structure, appropriate testing methods are required. Online weld quality monitoring $[1,2]$, such as visual inspection and infrared detection, and offline welding defects testing are the two main weld testing methods. Online monitoring method focus on weld puddle size, heat transfer rate, the working state of the welding machine (such as voltage, current, wire feed speed in arc welding machine), welding spatter, etc., while offline welding defects testing mainly concentrates on NDT (Non-Destructive Testing). Both methods have their own application scenarios. And the most widely used NDT methods are the ultrasonic method, radiographic method, magnetic method, penetrant method, electrical method, and acoustic emission method $[3,4]$.

Ultrasonic testing has the advantage of being able to detect flaws through thick materials, high sensitivities, and low cost. However, the defects are not displayed intuitively, ultrasonic inspection requires that the surface of the specimen to be smooth, and such testing requires highly experienced inspectors to recognize the defects. Radiographic detection can visually display the position of the defect in the direction of a plane, but cannot reflect the depth of a defect, and radiation causes damage to the human body. What's more, the recovery of the photochemical fixing solution is difficult and the direct emission and effluent can pollute the environment. Magnetic powder testing can only detect surface defects on ferromagnetic materials, and cannot detect the size of defects, where they are in the thickness of material, or their direction. Coloring or penetration testing methods are also proscribed to surface defects.

Weld flaw detection approaches based on vibration response analysis are also widely applied to online and offline detecting systems. Many researchers have contributed to establishing and evaluating these approaches. For example, both Shang [5] and Wang [6] studied how fatigue cracks impact the frequency domain of vibrations in spot welded specimens, and found that changes in natural frequencies and vibrating modes can be used to locate cracks. George Hearn [7] investigated the dependence of natural frequencies and modal damping coefficients on deterioration in structures using modal analysis. In addition, some other researchers [8,9] examined time-domain vibrating signals to monitor structures using methods based on HHT or wavelet transformation. Yang [10-12] introduced 
a concept of cross correlation function amplitude vector (CorV) based on acceleration response and proposed an approach to detect damages in complex structures. He conducted experiments on clamped beams and simple support boards to verify this approach. Dang [13] combined discrete wavelet transform (DWT) and CorV methods to detect damage in composite laminated structures. In the article, the author used DWT to remove the signal noise, detected the damages by comparing CorVs of responses obtained on intact and damaged structures, and verified the results by numeric simulations. In Lei's article [14], parameter $\mathrm{K}$, which is also based on CorV, was defined to describe the severity of damage in a steel frame structure model. Other methods related to the correlation coefficients between excitation and responses acquired on different points have been studied by Miao [15] and Wang [16,17]. In references [15-17], the authors located the damages by a sensor network and an approach of probability estimation.

The aim of this paper is to assess the potential of the CorV based on acceleration responses for detecting and locating welding defects. Our objective is to verify the ability of this method to locate defects and to detect the influence on CorV upon them. In order to achieve this aim, we simplify the simulation model into a flat butt plate with type-I welded joints recommended in GB985-88, as shown in Fig. 1.

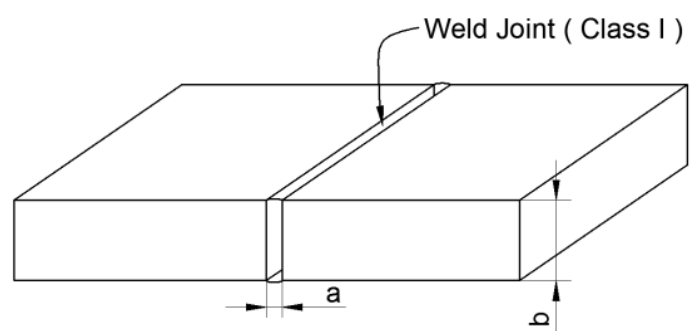

Figure 1. The parameters of welded joint (Type I)

In this paper, we study the influence of welded joint defects on correlation function amplitude vectors based on numerical simulations. First we construct a finite element model of a flat butt plate weld. Then we use methods based on CorV to find a discipline between the location of the defects and the corresponding changes of CorV acquired from responses in the numeric simulations. The structure of our paper is as follows: Section 2 presents the main principles of the CorV methods mentioned above. Section 3 shows the specific simulation structure and illustrates the results of numeric simulations and discussions. Section 4 concludes the paper and points out further work.

\section{The Simulation Structure and Principles of CorV Methods}

2.1 The Concept of CorV. In a linear elastic system, the motion can be described as the following differential equation

$$
[M]\{\ddot{y}\}+[C]\{\dot{y}\}+[K]\{y\}=[B]\{F\}
$$

In Eq. 1, the symbols $[M],[C]$, and $[K]$ represent the mass, damping and stiffness matrices. $[B]$ is the selection matrix, and $\{F\}$ is the input excitations. The symbol $y_{i}(t)$ represents the response acquired at the measuring point $i$. The solution of Eq. 1 in a given frequency domain is

$$
Y(\omega)=H(\omega) F(\omega)
$$

In Eq. 2, $H(\omega)$ is the frequency response function matrix of the structure. As is illustrated in Reference [10], the Cross Correlation Function Amplitude Vector is defined as the following equation.

$\Phi_{C o r V k}=\left\{\begin{array}{llll}\rho_{k 1} & \rho_{k 2} & \cdots & \rho_{k n}\end{array}\right\}$

In Eq. 3, parameter $k$ represents the freely selected reference point, and it is usually between excitation and measuring point. The element $\rho_{k i}$ represents the maximal value of the cross correlation function between response at reference point and response at measuring point, and can be calculated by solving the following equation

$$
\begin{aligned}
& \rho_{k i}=\max \left(R_{k i}(\tau)\right) \\
& R_{k i}(\tau)=\lim _{T \rightarrow \infty} \frac{1}{T} \int_{0}^{T} y_{k}(t) y_{i}(t+\tau) d t
\end{aligned}
$$


In Eq. 5, $y_{k}(t)$ represents the response at the reference point.

The time-averaged product [18] of $y_{k}(t)$ and $y_{i}(t)$ can be illustrated as follows:

$$
\begin{aligned}
& <y_{k}(t), y_{i}(t+\tau)>=\frac{1}{T} \int_{0}^{T} y_{k}(t) y_{i}(t+\tau) d t=\frac{1}{T} \int_{0}^{T} y_{k}(t)\left[\frac{1}{2 \pi} \int_{-\infty}^{\infty} Y_{i}(\omega) e^{j \omega(t+\tau)}\right] d t \\
& =\frac{1}{2 \pi} \frac{1}{T} \int_{0}^{T} Y_{i}(\omega) e^{j \omega \tau}\left[\int_{-\infty}^{\infty} y_{k}(t) e^{j \omega t} d t\right] d \omega=\frac{1}{2 \pi} \frac{1}{T} \int_{0}^{T} Y_{k}^{*}(\omega) Y_{i}(\omega) e^{j \omega \tau} d \omega
\end{aligned}
$$

Here, in Eq. 6, $Y_{k}^{*}(\omega)$ is the conjugate complex number of $Y_{k}(\omega)$. And we can obtain Eq. 7 from Eq. 2 and Eq. 6:

$$
<y_{k}(t), y_{i}(t+\tau)>=\frac{1}{2 \pi} \frac{1}{T} \int_{0}^{T}\left[H_{k}(\omega) F(\omega)\right]^{*}\left[H_{i}(\omega) F(\omega)\right] e^{j \omega \tau} d \omega
$$

When $T \rightarrow \infty$, Eq. 5 can be written as follows:

$R_{k i}(\tau)=E\left[y_{k}(t) y_{i}(t+\tau)\right]=\frac{1}{2 \pi} \int_{-\infty}^{\infty} \lim _{T \rightarrow \infty} E\left[\frac{1}{T} Y_{k}^{*}(\omega) Y_{i}(\omega)\right] e^{j \omega \tau} d \omega$

On substituting Eq. 2 into Eq. 8, the cross correlation function can be obtained by [10]

$R_{k i}(\tau)=\frac{1}{2 \pi} \int_{-\infty}^{\infty} \lim _{T \rightarrow \infty} E\left\{\frac{1}{T}\left[H_{k}(\omega) F(\omega)\right]^{*} \bullet\left[H_{i}(\omega) F(\omega)\right]\right\} e^{j \omega \tau} d \omega$

From Eq. 9, we can conclude that the cross correlation function is decided by the excitation and the frequency response function matrix. And when the excitation is constant, the CorV of the structure depends only on FRF matrix. If there is any damage that appears in the structure, the mass, damping or stiffness matrix will be affected, and that influence will have an impact on FRF matrix and CorV. In other words, when the excitation is specific, the CorV is only related to the response at the reference point and the measuring point.

In order to facilitate the observation, the value of $\Phi_{C o r V_{k}}$ is normalized by the following equation

$\hat{\Phi}_{C o r V_{k}}=\left\{\frac{\rho_{k i}}{\sqrt{\sum \rho_{k j}^{2}}}\right\}$

The criteria for discriminating defects can be defined as the following equation

$$
I_{c o r V}=\frac{\left|\hat{\Phi}_{C o r V_{N C}}-\hat{\Phi}_{C o r V_{k}}\right|}{\hat{\Phi}_{C o r V_{N C}}}
$$

Here, $\hat{\Phi}_{C o r V_{N C}}$ and $\hat{\Phi}_{C o r V_{k}}$ represent the cross correlation function vector from intact welded joints and damaged welded joints respectively. And the defects in welded joints can be reflected by relative change of CorV, which is described as $I_{\text {cor } V}$.

2.2 The Finite Element Model of the Welded Joints Structure. In order to verify that CorV can be applied to the detection of welded joints, a finite element model of a clamped board has been established, and it is shown in Fig. 2.

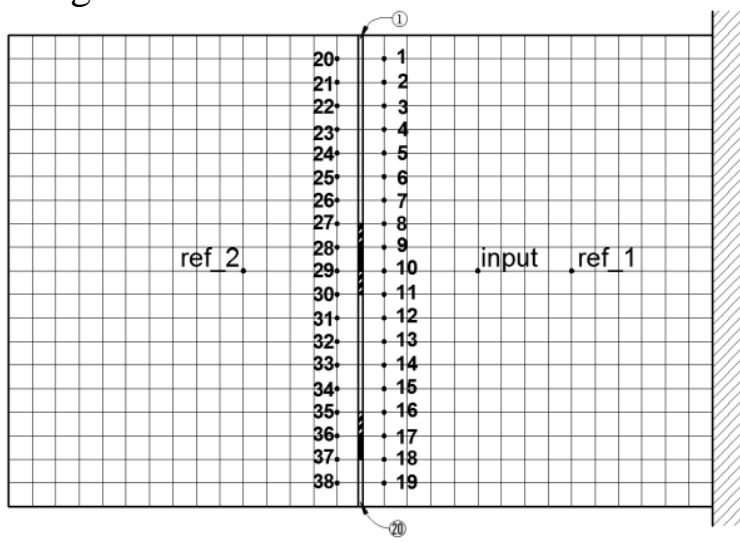

Figure 2. The finite element model of the welded joint structure

The main dimensions of the model are: $300 \mathrm{~mm}$ long, $200 \mathrm{~mm}$ wide, and $2 \mathrm{~mm}$ thick. The model is divided into $30 \times 20$ grids, and each grid is meshed into 25 hexahedral elements. The welded joint is 
represented with the middle area in Fig. 2, and it is $2 \mathrm{~mm}$ wide. We divide it into 20 grids, and number the grids in sequence as is shown in Fig. 2. Each grid is meshed into 5 elements. The density of the board and welded joint is $7800 \mathrm{~kg} / \mathrm{m}^{-3}$. The modulus of elasticity and the Poisson's ratio of the board is $206 \mathrm{GPa}$ and 0.23 respectively. As is known to all, the strength of welded joint without defects is greater than base material. Therefore, considering the influence of the welded joint, the modulus of elasticity of welded joint is set as $235 G P a$.

\section{Numeric Simulation}

In this section, we report on numeric simulations we performed to verify the feasibility of the welded defects detection method based on CorV. In this study, we used the model established above to find some disciplines between the location of welded defects and CorVs, and also the relation between CorVs and severity of the defects. The excitation force shown in Fig. 3 is a 3 -cycle sine wave modulated by Hanning window, and it is applied at the input point shown in Fig. 2. It is also shown in Fig. 2 that the measuring point located on both sides of the welded joint numbers from 1 to 38 , and the distance between welded joint and measuring point is $9 \mathrm{~mm}$. In addition, we set two reference points to confirm that the position of the reference point has no effect on the identification of defects based on $I_{c o r V}$. All the defects we studied are simulated by reducing the modulus of elasticity of damaged elements. All of the responses acquired are accelerations in $0.0001 \mathrm{~s}$. Velocity and displacement responses can also be used to identify where the defects are.

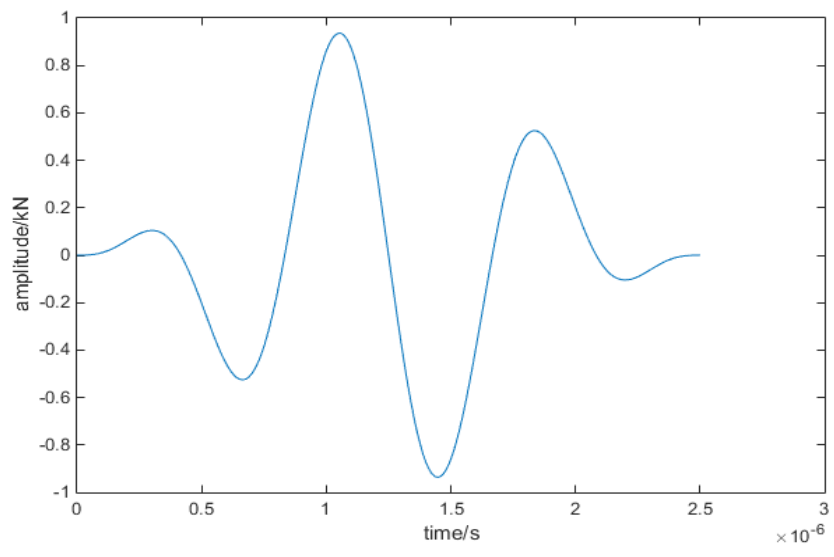

Figure 3. The excitation force in time domain

3.1 The Influence of Defects Location. We set five conditions to study the influence of each defect's location, and each condition with a 95\% reduction in modulus of elasticity is shown in Table I.

Table I. Five Conditions of Different Defects Location
\begin{tabular}{|c|c|c|c|c|c|}
\hline Conditions & A & B & C & D & E \\
\hline $\begin{array}{l}\text { Element } \\
\text { Number }\end{array}$ & 9 & 10 & 11 & 17 & 9 \& 18 \\
\hline
\end{tabular}

The simulation results of different conditions using reference one (ref_1) are shown in Fig. 4. In these simulations, four conditions (A D) with single damaged welded joint element and one condition (E) with double damaged welded joint elements have been considered. When there is no defect between input and measuring points or reference points, the responses acquired at both measuring and reference points must have some relationship, because the input and the structure is certain, which means that $H(\omega)$ is certain. If there are defects occurring between measuring and input points, the relations between the responses acquired at measuring points and reference points must be changed. The position of welded joint defects can be identified from Fig. 4, especially the defects in the middle of the board. In condition A, $I_{c o r V}$ at point 27 is the maximum value which indicates that the response at point 27 changes drastically comparing with intact welded joint. In condition $\mathrm{B}$ and $\mathrm{C}$, the biggest changes are at point 28 and point 30 respectively. A line can be drawn between input point and measuring point, and the line cross element 9 joins point 27 and the input point. Therefore, if element 9 is damaged, the acquired signal will be different, and this will make the $I_{c o r V}$ the maximum value. 


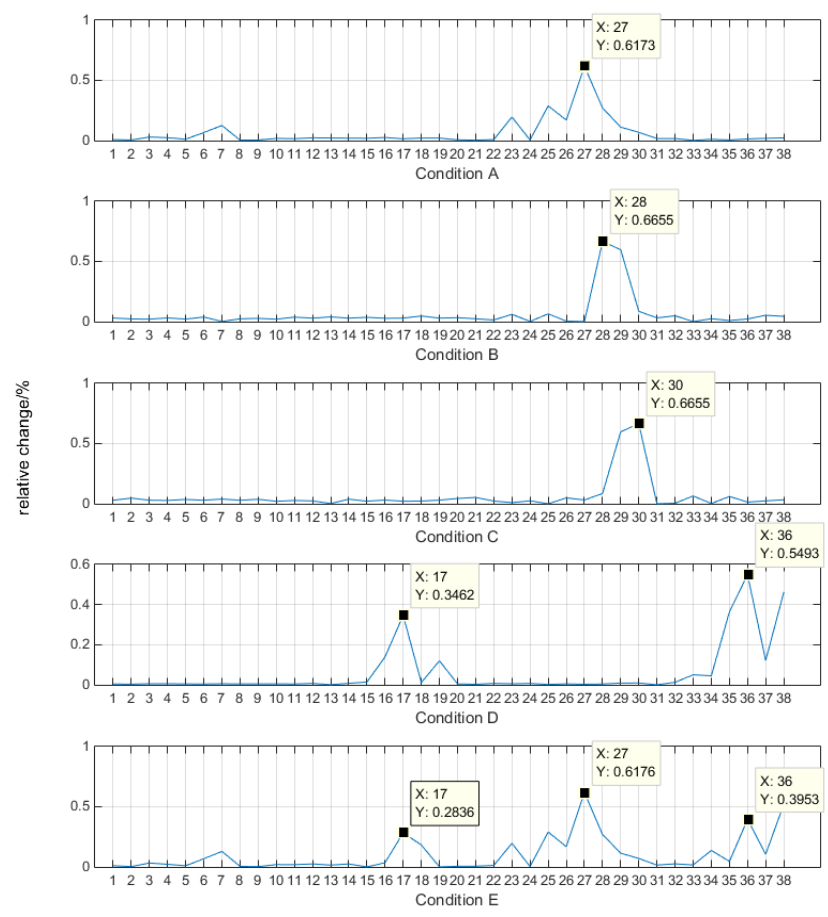

Figure 4. The Map of Measuring Points and Relative Change of CorV in Different Conditions Using Reference Point One (ref_1)

In condition 4, line connecting point 36 and the input point passes through element 17 . Therefore, welded defects can also be identified. However, because of the influence of boundary reflection responses, defects close to boundaries may be hard to identify. Reference [19] has already investigated this problem using HHT method, cubic curve fitting method, and extension method.

In condition 5, responses at point 27,36, 17 and 38 change relatively intensely, because of the existence of defects in element 9 and 18. In other words, double defects can also be identified by this method. The same results will be found if we calculate $I_{\text {corV }}$ by choosing point ref_2 in Fig. 2 as reference points. The results are shown in Fig. 5.

3.2 The Influence of Severity of Defects. In order to investigate the influence of the severity of defects in one welded joint element, we reduce the modulus of elasticity by $10-80 \%$, and the damaged element is No. 9. The results are shown in Fig. 6 and Fig. 7.
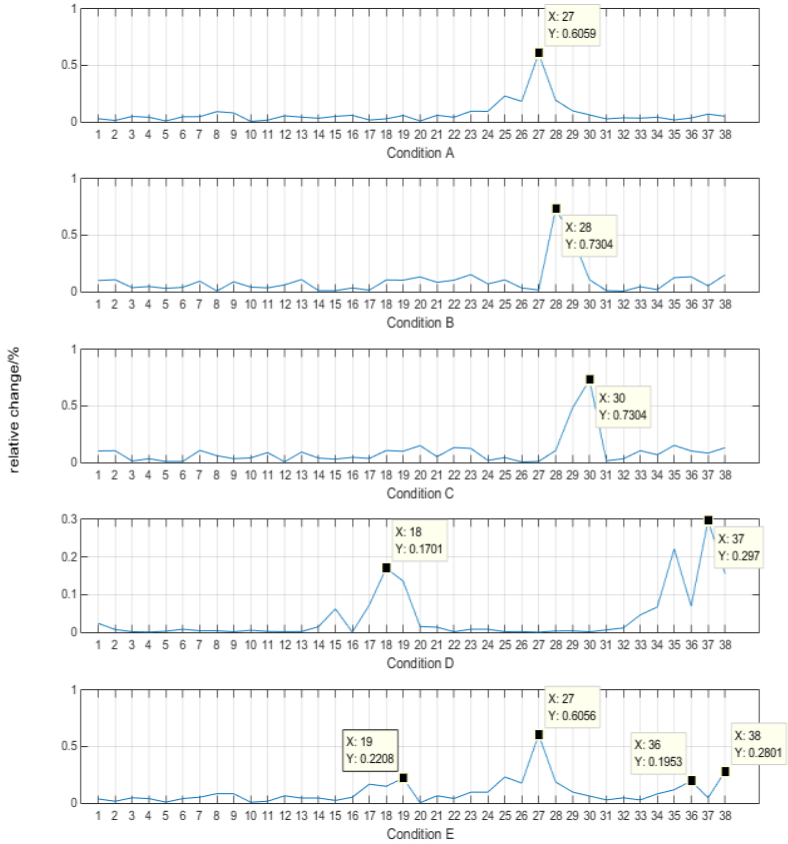

Figure 5 The map of measuring points and relative change of CorV in different conditions using reference point two (ref_2)

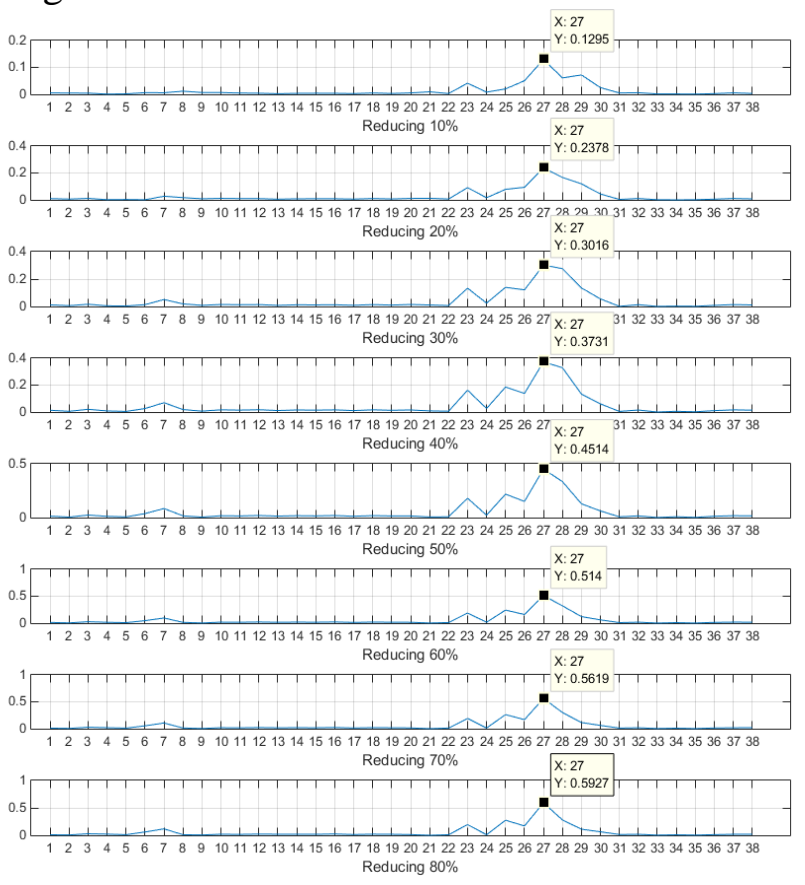

Figure 6 The map of measuring points and relative change of CorV in the element 9 with different severity of defects 
As the results shown in Fig. 6, we can confirm again that welded joint defects in a single element can be identified by this method, and it has stable performance with high accuracy of identification. And the disturbances at point 7 and point 8 are caused by the defects.

To find a discipline between the amount decreases in modulus of elasticity and the relative change of CorV $-I_{c o r V}$, the maximum value of each set of data is extract, and the results of the relationship between defect severity and relative change in $I_{c o r V}$ are shown in Fig. 7. We use three polynomials to fit these points, and get an equation shown as follows:

$$
y(x)=-2.911 * 10^{-7} x^{3}-2.987 * 10^{-6} x^{2}+0.008951 x+0.04717
$$

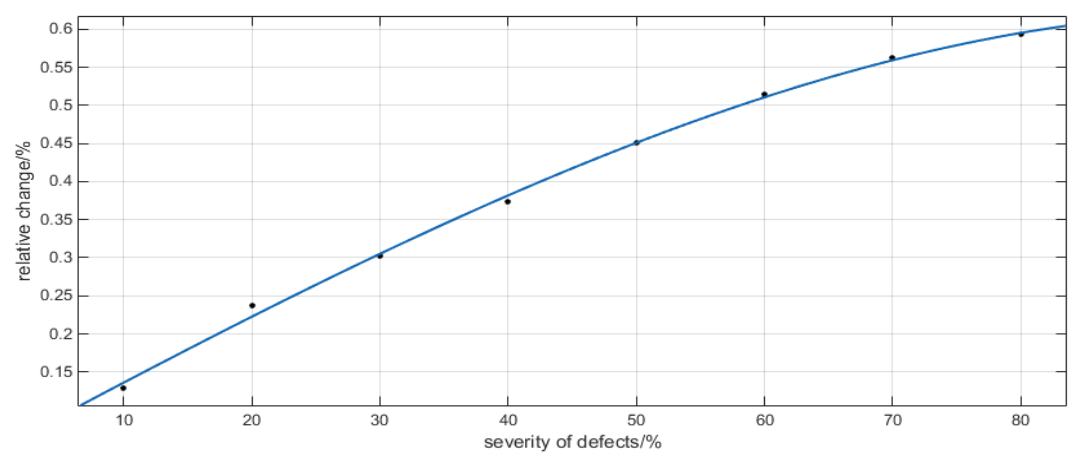

Figure 7 The relative change of CorV in the element 9 with different severity of defects

In Eq. 12, $x$ represents the percent reduction in modulus of elasticity, and $y(x)$ represents the percentage of $I_{\text {corV }}$.

When the severity of defects is $95 \%$, the error between the calculation results by Eq. 11 and the measuring result shown in Fig. 4 is about $0.599 \%$. In conclusion, Eq. 11 has a certain accuracy, and $I_{c o r V}$ increases as the severity of the defects increases.

\section{Conclusions}

In this paper, we verify that the damage detection method based on the cross correlation function amplitude vector can be applied in the identification of weld defects in flat butt plate with type-I welded joints. We also studied the influence and severity of defects. We conclude that the existence of welded joint defects between input point and measuring point can influence greatly these responses. Furthermore, the relative change of CorV increases as the severity of the defects increases and the relationship follows the cubic polynomial equation. Compared with traditional NDT, this method is much more convenient and the results can be calculated quickly. In this paper, we investigate the influence of defects based on numeric simulation. Further experiments need to be performed. And the influences of multi-defects on welding structures should be examined in further work.

\section{Acknowledgment}

This work was sponsored by Natural Science Foundation of Shanghai (No.16ZR1408400).

\section{References}

[1] Miller, Matthew. 2002. Development of automated real-time data acquisition system for robotic weld quality monitoring, Mechatronics, 12(9-10): 1259-1269.

[2] Y. Li. 2010. Measurement and Defect Detection of the Weld Bead Based on Online Vision Inspection, IEEE Transactions on Instrumentation \& Measurement, 7(59): 1841-1849.

[3] R. Halmshaw. 1988. Introduction to the Non-Destructive Testing of Welded Joints, Gamma Rays.

[4] R. J. Ditchburn, S. K. Burke, C. M. Scala. 1996. NDT of welds: state of the art, NDT \& E International, 29(96): 111-117. 
[5] Shang. 2003. Fatigue Damage and Dynamic Natural Frequency Response of Spot-Welded Joints, Sae Technical Papers, (112): 301-308.

[6] G. Wang and M. E. Barkey. 2004. Fatigue cracking and its influence on dynamic response characteristics of spot welded specimens, Experimental Mechanics, 5(44): 512-521.

[7] Hearn, George and R. B. Testa. 1991. Modal Analysis for Damage Detection in Structures, Journal of Structural Engineering, 10(117): 3042-3063.

[8] Rezaei, Davood and F. Taheri. 2010. Health monitoring of pipeline girth weld using empirical mode decomposition, Smart Materials \& Structures, 5(19): 0055016.

[9] M. Rucka and K. Wilde. 2006. Application of continuous wavelet transform in vibration based damage detection method for beams and plates, Journal of Sound \& Vibration, 3(297): 536-550.

[10]Z. C. Yang, Z. Yu and H. Sun. 2006. On the cross correlation function amplitude vector and its application to structural damage detection, Mechanical Science \& Technology, 7(21): 2918-2932.

[11]Z. C. Yang. 2008. Damage detection fo composite structures by a hybrid method combing CorV and wavelet transform, Journal of Vibration and Shock, 27(11): 17-21.

[12]Z. C. Yang. 2008. Experimental Research on Detection in Fastener-Loosing of Aircraft Panel by Correlation Function, Chinese Journal of Applied Mechanics, 25(1): 99-102.

[13] X. J. Dang. 2010. Damge detction of composite laminated structrue by discrete wavelet transform and CorV method, Journal of Mechanical Strength, 2(32): 202-206.

[14]J. Y. Lei. 2011. Structural damage detection method based on correlation function analysis of vibration measurement data, Journal of Vibration of Shock, 30(8): 221-224,236.

[15]X.T. Miao, J.J. Zhao, F.C. Li, F.G. Zhang and G. Meng. 2016. A Damage Location Method Based on The Correlation Coefficient of Energy Spectrum for Wave Signals, Journal of Mechanical Engineering, 52(15): 73-80.

[16]D. Wang. 2010. A damage diagnostic imaging algorithm based on the quantitative comparison of Lamb wave signals, Smart Materials \& Structures, 19(19): 065008.

[17]D. Wang. 2009. Probability of the presence of damage estimated from an active sensor network in a composite panel of multiple stiffeners, Composites Science and Technology, 69(13): 2054-2063.

[18]D. J. Ewins. 1985. Modal Testing: Theory and Practice, New York: Wiley.

[19]Z. P. Shu. 2006. Investigation of Structural Damage Detection Based on Hilber-Huang Transformation, Northwestern polytechnical university. 